\title{
Lactobacillus Causing Urinary Tract Infection in a Neonate
}

\author{
Citla Sridhar Divyaswathi ${ }^{\mathrm{a}}$, Anand Gourishankar ${ }^{\mathrm{b}, \mathrm{c}}$
}

\begin{abstract}
Except for their involvement in dental caries, lactobacilli are usually considered to be non-pathogenic, despite occasional reports of serious infections possibly secondary to underlying predisposing conditions like immunodeficiency, malabsorption syndromes, indwelling catheters and malignancy. Here we report a rare case of urinary tract infection (UTI) in neonate due to Lactobacillus species. Lactobacillus causing UTI in our patient is convincing since the Lactobacillus species was isolated in significant quantities from a catheterized urine sample and responded to treatment. The pathogenesis of this infection in our patient is intriguing yet interesting. The fact that she was not breast fed, female gender and a neonate with diminished immune function may have played a role in lactobacillus urinary tract infection. To the best of our knowledge, however, UTI caused by Lactobacillus species in a neonate have not been reported previously.
\end{abstract}

Keywords: Lactobacillus; Urinary tract infection; Neonate

\section{Introduction}

Urinary tract infection (UTI) is more prevalent in late onset sepsis compared to early onset infection in infants. Virulence of the pathogen, age, race, functional and anatomical risk factors in children are published and poorly understood. Lactobacillus is not implicated in both community acquired and nosocomial neonatal UTI and there are cases of lactobacillus bacteremia in 8 - 96 years old who are either immunosuppressed or had catheters [1]. Here we report this rare case of

\footnotetext{
Manuscript accepted for publication August 12, 2013

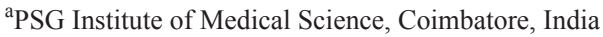

${ }^{\mathrm{b}}$ Division of Community and General Pediatrics, University of Texas

Medical School at Houston, Texas, USA

${ }^{\mathrm{c} C}$ Corresponding author: Anand Gourishankar, 6431 Fannin Street,

Room JJL 495, Houston 77030 Texas, USA.

Email: anand.gourishankar@uth.tmc.edu
}

doi: http://dx.doi.org/10.4021/jmc1454w
UTI in neonate due to Lactobacillus species.

Most of these are caused by gram negative aerobic bacilli / Enterobacteriaceae, the commonest being due to Escherichia coli. Neonatal UTI is more common in boys, especially uncircumcised and it is associated with bacteremia [2].

However reports of Lactobacillus species causing UTI in an infant are very scarce in the literature.

\section{Case Report}

A 4-week-old African American girl, born at term, with no perinatal complications, presented with fever. One week prior to presentation, she developed fever, fussiness and vomiting after feeds. She was diagnosed to have colic at an outside hospital and sent home. She continued to have gradual worsening of symptoms, with intermittent fever, decreased frequency of urination, increasing fussiness and multiple episodes of non-bilious and non-bloody vomiting. She was on formula feeds, had no recent sick contacts and was cared at home.

The child was born as a result of a normal vaginal delivery at 38 weeks after induction of labor. There was no history of Group B Streptococcus (GBS) or herpes infection in the mother during pregnancy. Postpartum period was uneventful.

At admission, weight was 3.95 (61st \%), length was 55 $\mathrm{cm}(90$ th \%), head circumference was $37 \mathrm{~cm}$ (75th \%). On examination, the child had a temperature of $38.7^{\circ} \mathrm{C}$, respiratory rate of 46 breaths per minute, heart rate of 152 beats per minute and oxygen saturation of $99 \%$ on room air. She was awake, alert, and irritable. Her pupils were equally round and reactive to light. The cardiac exam was normal and lungs were clear with breath sounds bilaterally. Abdomen was soft with no distension or tenderness, and bowel sounds were heard. She appeared slightly dehydrated with a capillary refill time of 4 seconds. The neurological exam revealed no deficit. A papular, non-blanching, non-erythematous, nonvesicular rash was noted at the back of the neck.

A complete blood count revealed white blood cell count of 12,500 cells $/ \mathrm{mm}^{3}$, hemoglobin of $10.6 \mathrm{~g} / \mathrm{dL}$ and platelets of $396,000 / \mathrm{mm}^{3}$. The differential count was as follows: Seg- 
mented Neutrophils 20\%, Lymphocytes 63\%, Monocytes 10.0\%, Eosinophil 5.0\%, and Basophils 1.0\%.

The metabolic panel revealed: sodium of $138 \mathrm{mEq} / \mathrm{L}$, blood urea nitrogen of $7 \mathrm{mg} / \mathrm{dL}$, creatinine of $0.5 \mathrm{mg} / \mathrm{dL}$ and blood glucose of $94 \mathrm{mg} / \mathrm{dL}$. Urinalysis showed specific gravity 1.015 , blood trace, ketones negative, protein $30 \mathrm{mg} / \mathrm{dl}$, urobilinogen 0.2 , leukocyte esterase moderate, nitrites negative, bacteria few, reducing substances negative.

She was started empirically on meningitic doses of ampicillin and cefotaxime. Blood cultures were negative and her urine culture from a catheterized urine sample revealed $>100,000 \mathrm{CFU} / \mathrm{ml}$ of lactobacillus species, sensitive to ampicillin, vancomycin and cefotetan. Hence a diagnosis of UTI secondary to Lactobacillus infection was made. The rash on the back of neck was possibly due to contact dermatitis. Infant completed 14 days of intravenous ampicillin without any complications.

\section{Discussion}

UTI is a serious infection usually affecting kidneys and associated with bacteremia or urosepsis in neonates and pooled prevalence in $<3$ months with fever is $7.2 \%(5.8-8.6)$ [3]. In a prospective study, infants aged 0 to 3 months represented $21 \%$ of children diagnosed with first UTI, suggesting that UTI may be a relatively common cause of fever in these patients [4] . Most UTIs in infants are caused by Gram negative aerobic bacilli known as Enterobacteriaceae. Escherichia coli are the most common organisms isolated in the newborn period, accounting for up to $80 \%$ of infections in most large series studies. Other Enterobacteriaceae, which can produce UTI, include: Klebsiella, Enterobacter, Citrobacter, Proteus, Providencia, Morganella, Serratia, and Salmonella species $[5,6]$.

Lactobacillus species are non-sporing, gram positive, facultative anaerobic rod. Lactobacillus species are commensals of the human mucosal tissues, including oropharynx, vagina and the gut $[7,8]$. Except for their involvement in dental caries lactobacilli have usually been considered to be non-pathogenic, despite occasional reports of serious infections possibly associated with them over several decades [9-11]. In the past, lactobacilli isolated from human infections were often dismissed as contaminants, secondary invaders or misidentified as streptococci [12].

However, there is now increasing evidence that they can act as opportunistic pathogens [13]. Clinical syndromes associated with Lactobacillus include dental caries and periodontal abscesses, intra-abdominal abscess, bacteremia as a part of polymicrobial process, endocarditis, urinary tract infection in women, chorioamnionitis/endometriosis [7]. There are two case reports of Lactobacillus species causing UTI in older adult females which suggest that that in select situations, Lactobacillus spp. should not be regarded simply as a contaminant but as an unlikely, yet significant, cause of UTI in an otherwise immune competent female patients $[14,15]$.

Lactobacillus bacteremia among children is very unusual and only a very few cases have been reported, primarily among immunocompromised hosts [16, 17]. The underlying conditions include acquired immunodeficiency syndrome, immunosuppression after bone marrow transplantation for treatment of aplastic anemia and dental abscesses [18]. In a study, Lactobacillus species were isolated in 40 children, aged between 8 months and 16 years, where most isolates were from abscesses, aspiration pneumonia, ear infections, bacteremia and conjunctivitis. Infections were mostly polymicrobial. Four Lactobacillus isolates from blood cultures were in pure culture and were from four patients with intravenous catheter-related bacteremia. Eight of the patients had one or two predisposing or underlying medical or surgical conditions like intravenous catheter, immunodeficiency, malabsorption or malignancy [19].

There is a possibility of acquiring Lactobacillus infections from probiotic use. Serious infections due to probiotic lactobacilli are extremely rare. Two adult cases have been reported in which the Lactobacillus rhamnosus strain isolated from the clinical specimen was indistinguishable from the probiotic strain consumed by the patient $[20,21]$. There are case reports where a six-week-old infant and a six-year-old girl were treated for a presumed antibiotic associated diarrhea with oral Lactobacillus containing probiotics and had later yielded positive blood cultures of Lactobacillus species, which by DNA fingerprinting analysis, were found to be indistinguishable from the probiotics strains prescribed to the patients [18]. Lactobacillus rhamnosus GG bacteremia and probiotic use in children with short gut syndrome is also reported [22].

On the brighter side probiotic use is recommended for various pediatric medical conditions [23]. Six year old girl with normal renal anatomy with third recurrent UTI improved with probiotic [24]. Prophylactic probiotics use was no different compared to antibiotic prophylaxis in primary vesicoureteral reflux [25]. Very low birth weight preterm infant benefitted with probiotic with decreased mortality and necrotizing enterocolitis [26].

Lactobacillus causing UTI in our patient is convincing since the Lactobacillus species was isolated in significant quantities from a catheterized urine sample and responded to treatment. It is difficult to discount it as a contamination.

The pathogenesis of this infection in our patient is intriguing yet interesting. There is neither prior antibiotic/probiotic use nor central line placement. Her renal ultrasound and voiding cystourethrogram was normal as well.

However, in the microbiological diagnosis of UTI, lactobacilli plays a certain role as pathogens of non-specific cysto-urethritis, which only occurs as a result of excessive colonization [14]. 
The imbalance between uropathogens and normal flora has been suggested to be a risk factor for UTI. Lee et al did a case control study where Lactobacillus culture was low in stool, peri-uretheral swab and urine compared to vaginal swab in infants less than 1year old with UTI. Less urogenital lactobacillus colonization may be a risk factor for UTI in infants [27]. However, if excessive colonization itself causes poses as a risk factor is not known. According to a study, very small percentages $(1.7 \%)$ of cases of endocarditis with Lactobacillus infections were associated with heavy dairy consumption [28].

We could not identify obvious risk factors in this case but could not ignore the fact she was not breast fed, female gender, a neonate with diminished immune function may have played a role in lactobacillus UTI.

\section{Conflict of Interest}

No conflict of interest or disclosures to report for both authors.

\section{References}

1. Gouriet F, Million M, Henri M, Fournier PE, Raoult D. Lactobacillus rhamnosus bacteremia: an emerging clinical entity. Eur J Clin Microbiol Infect Dis. 2012;31(9):2469-2480.

2. Beetz R. Evaluation and management of urinary tract infections in the neonate. Curr Opin Pediatr. 2012;24(2):205-211.

3. Shaikh N, Morone NE, Bost JE, Farrell MH. Prevalence of urinary tract infection in childhood: a meta-analysis. Pediatr Infect Dis J. 2008;27(4):302-308.

4. Ismaili K, Lolin K, Damry N, Alexander M, Lepage P, Hall M. Febrile urinary tract infections in 0 - to 3-monthold infants: a prospective follow-up study. J Pediatr. 2011;158(1):91-94.

5. Maherzi M, Guignard JP, Torrado A. Urinary tract infection in high-risk newborn infants. Pediatrics. 1978;62(4):521-523.

6. Honkinen O, Lehtonen OP, Ruuskanen O, Huovinen P, Mertsola J. Cohort study of bacterial species causing urinary tract infection and urinary tract abnormalities in children. BMJ. 1999;318(7186):770-771.

7. Antony SJ. Lactobacillemia: an emerging cause of infection in both the immunocompromised and the immunocompetent host. J Natl Med Assoc. 2000;92(2):83-86.

8. Darbro BW, Petroelje BK, Doern GV. Lactobacillus delbrueckii as the cause of urinary tract infection. J Clin Microbiol. 2009;47(1):275-277.

9. Sharpe ME, Hill LR, Lapage SP. Pathogenic lactobacilli. J Med Microbiol. 1973;6(3):281-286.
10. Biocca E, Seppilli A. Human infections caused by lactobacilli. J Infect Dis. 1947;81(2):112-115.

11. Dietzsch HJ. [Subacute bacterial endocarditis caused by lactobacilli in child]. Monatsschr Kinderheilkd. 1955;103(4):240-243.

12. Swenson JM, Facklam RR, Thornsberry C. Antimicrobial susceptibility of vancomycin-resistant Leuconostoc, Pediococcus, and Lactobacillus species. Antimicrob Agents Chemother. 1990;34(4):543-549.

13. Aguirre M, Collins MD. Lactic acid bacteria and human clinical infection. J Appl Bacteriol. 1993;75(2):95-107.

14. Dickgiesser U, Weiss N, Fritsche D. Lactobacillus gasseri as the cause of septic urinary infection. Infection. 1984;12(1):14-16.

15. Bernier M, Njomnang Soh P, Lochet A, Prots L, Felice R, Senescau A, Fabre R, et al. [Lactobacillus delbrueckii: probable agent of urinary tract infections in very old women]. Pathol Biol (Paris). 2012;60(2):140142.

16. Schlegel L, Lemerle S, Geslin P. Lactobacillus species as opportunistic pathogens in immunocompromised patients. Eur J Clin Microbiol Infect Dis. 1998;17(12):887888.

17. Robin F, Paillard C, Marchandin H, Demeocq F, Bonnet R, Hennequin C. Lactobacillus rhamnosus meningitis following recurrent episodes of bacteremia in a child undergoing allogeneic hematopoietic stem cell transplantation. J Clin Microbiol. 2010;48(11):4317-4319.

18. Land MH, Rouster-Stevens K, Woods CR, Cannon ML, Cnota J, Shetty AK. Lactobacillus sepsis associated with probiotic therapy. Pediatrics. 2005;115(1):178-181.

19. Brook I. Isolation of non-sporing anaerobic rods from infections in children. J Med Microbiol. 1996;45(1):2126.

20. Rautio M, Jousimies-Somer H, Kauma H, Pietarinen I, Saxelin M, Tynkkynen S, Koskela M. Liver abscess due to a Lactobacillus rhamnosus strain indistinguishable from L. rhamnosus strain GG. Clin Infect Dis. 1999;28(5):1159-1160.

21. Mackay AD, Taylor MB, Kibbler CC, Hamilton-Miller JM. Lactobacillus endocarditis caused by a probiotic organism. Clin Microbiol Infect. 1999;5(5):290-292.

22. De Groote, M. A., Frank, D. N., Dowell, E., Glode, M. P. \& Pace, N. R. Lactobacillus rhamnosus GG bacteremia associated with probiotic use in a child with short gut syndrome. Pediatr. Infect. Dis. J. 2005; 24 (3) : 278-280.

23. Thomas DW, Greer FR. Probiotics and prebiotics in pediatrics. Pediatrics. 2010;126(6):1217-1231.

24. Gerasimov SV. Probiotic prophylaxis in pediatric recurrent urinary tract infections. Clin Pediatr (Phila). 2004;43(1):95-98.

25. Lee SJ, Shim YH, Cho SJ, Lee JW. Probiotics prophylaxis in children with persistent primary vesicoureteral reflux. Pediatr Nephrol. 2007;22(9):1315-1320. 
26. Lin HC, Hsu CH, Chen HL, Chung MY, Hsu JF, Lien RI, Tsao LY, et al. Oral probiotics prevent necrotizing enterocolitis in very low birth weight preterm infants: a multicenter, randomized, controlled trial. Pediatrics. 2008;122(4):693-700.

27. Lee JW, Shim YH, Lee SJ. Lactobacillus colonization status in infants with urinary tract infection. Pediatr Nephrol. 2009;24(1):135-139.

28. Cannon JP, Lee TA, Bolanos JT, Danziger LH. Pathogenic relevance of Lactobacillus: a retrospective review of over 200 cases. Eur J Clin Microbiol Infect Dis. 2005;24(1):31-40. 